\title{
DESIGN OF A FUZZY GAIN SCHEDULING CONTROLLER HAVING INPUT SATURATION: A COMPARATIVE STUDY
}

\author{
Yan-Wen Huang \\ Department of Mechanical Engineering, Nanya Institute of Technology, Taoyuan County, Taiwan, R.O.C. Department of \\ Mechanical Engineering, National Central University, Taoyuan County, Taiwan, R.O.C., hyw@nanya.edu.tw \\ Pi-Cheng Tung \\ Department of Mechanical Engineering, National Central University, Taoyuan County, Taiwan, R.O.C.
}

Follow this and additional works at: https://jmstt.ntou.edu.tw/journal

Part of the Mechanical Engineering Commons

\section{Recommended Citation}

Huang, Yan-Wen and Tung, Pi-Cheng (2009) "DESIGN OF A FUZZY GAIN SCHEDULING CONTROLLER HAVING INPUT SATURATION: A COMPARATIVE STUDY," Journal of Marine Science and Technology: Vol. 17: Iss. 4, Article 1. DOI: $10.51400 / 2709-6998.1980$

Available at: https://jmstt.ntou.edu.tw/journal/vol17/iss4/1

This Research Article is brought to you for free and open access by Journal of Marine Science and Technology. It has been accepted for inclusion in Journal of Marine Science and Technology by an authorized editor of Journal of Marine Science and Technology. 


\title{
DESIGN OF A FUZZY GAIN SCHEDULING CONTROLLER HAVING INPUT SATURATION: A COMPARATIVE STUDY
}

\author{
Yan-Wen Huang**** and Pi-Cheng Tung**
}

\begin{abstract}
Key words: fuzzy gain scheduling, proportional controllers, input saturation, adaptive control, fuzzy PD system, describing function, Nyquist, linear quadratic regulator (LQR).
\end{abstract}

\begin{abstract}
A fuzzy gain scheduling proportional controllers that exhibits the improved performance than the conventional linear fixed gain controller having input saturation is proposed in this paper. We proposed an adaptive anti-windup control system in which the gain of a proportional controller is dynamically tuned by a fuzzy PD system. The methodology employed in the analysis is based on the describing function (DF), which is determined the range of the universal discourse for the output of the fuzzy PD system. Other approaches are the linear quadratic regulator (LQR) method and combined back-calculation and generalized conditioning anti-windup. In this paper, a comparison study of these three approaches for design anti-windup controller is made. The simulation results show the differences and confirm the availability of the proposed design approaches.
\end{abstract}

\section{INTRODUCTION}

Proportional controllers have been the most popular and the most commonly used industrial controllers in the past years. Although linear fixed gain proportional controls are often adequate for controlling a nominal physical process, the requirements for high-performance control with changes in operating conditions or environmental parameters are often beyond the capabilities of simple proportional controllers. In order to improve the performance of the linear proportional controllers, this paper presented an adaptive fuzzy gain scheduling proportional controllers by a fuzzy PD reasoning mechanism system.

In a control system, modeling errors, and control input satu-

Paper submitted 10/17/06; revised 02/09/07; accepted 05/03/07. Author for correspondence: Yan-Wen Huang (e-mail: hyw@nanya.edu.tw).

*Department of Mechanical Engineering, Nanya Institute of Technology, Taoyuan County, Taiwan, R.O.C.

**Department of Mechanical Engineering, National Central University, Taoyuan County, Taiwan, R.O.C. ration are unavoidable. The term "windup" originates from the tendency of integral controllers to "windup" under input saturation conditions. Control input saturation in the system can lead to severe transient oscillations and a large overshoot. Several compensator designs have been proposed to address the windup problem (e.g. Edward and Postlethwaite [3], Astrom and Rundqwist [1]). Several researchers have employed absolute stability theory to guarantee the closed loop stability of multivariable systems in the presence of input nonlinearities (e.g. Saeki and Wada [11], Turner and Postlethwaite [12]). Recently, a conceptually simple approach to fuzzy control design has been proposed for nonlinear systems with actuator saturation (Hansson et al. [6]; Gharieb and Nagib [5]; Zhao and Collins [13]).

The term "windup" systems from the tendency of integral controllers to "windup" during control input saturation. However, the "windup phenomenon" degrades the performance of controlled systems, resulting in large overshoots, long settling times, and, in severe cases, severe transient oscillations, and system instability. Since these undesired effects of plant input saturation can be attributed to the controller, they are called "controller windup". If the closed-loop dynamics assigned by the control are "too fast", large overshoots, long settling times, or even instability can occur which dynamics of the controlled plant, referred to as "(oscillatory) plant windup" (Hippe [7]). In this paper an adaptive fuzzy gain scheduling proportional controllers in which the controller gains on line is dynamically tuned by a fuzzy PD reasoning mechanism system is proposed. The range of the output of universal discourse for the output of the fuzzy PD system is designed based on the describing function stability criterion to assume system's stability. The fuzzy adaptation algorithm "plant windup" scheme will be used to utilize not only the control error but also the control signal saturation for the modification control input signal. The proposed controller not only prevents "controller windup", but also modifies the control input signal to prevent "plant windup". Other approaches are the linear quadratic regulator (LQR) method and combined back-calculation and generalized conditioning anti-windup. In this paper, a comparison study of these three approaches for design anti-windup controller is made. The differences in the performance of the three methods are then studied. 


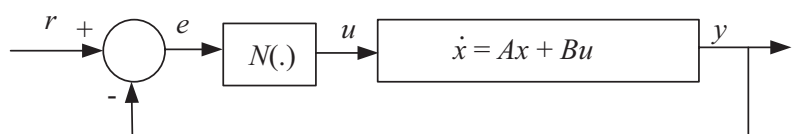

Fig. 1. Fuzzy adaptive control systems having input saturation plant anti-windup.

The remainder of this paper is organized as follows. Section II presents stability analysis based on describing function having input saturation. Section III presents the fuzzy PD system adaptive gain tuning controller design methods. Section IV simulation studies of a linear system with saturating input nonlinearity indicates that the application of the proposed anti-windup approach and compares the resulting system response to that obtained using the LQR anti-windup approach. Finally, Section V summarizes the conclusions.

\section{STABILITY ANALYSIS}

This study considers a control system having control input saturation as shown in as shown in Fig. 1, which illustrates a 'standard' nonlinear control system configuration, consisting of a nominal system with a transfer function $\mathrm{G}(\mathrm{s})$, preceded by a single, static (i.e., "memoryless" nonlinear element N( .) ).

The following expressions for nonlinear system described by:

$$
\begin{gathered}
\dot{x}=A x+B u, \\
y=C x, \\
e=r-y, \text { (tracking error), } \\
u=N(.)
\end{gathered}
$$

where $x \in R^{n}$ is an n-dimensional state. Furthermore, $r \in R$ is the desired trajectory; $u \in R$ is the control input; $y \in R$ is the output; $e \in R$ is the tracking error; $k$ denotes the gain of the controller, $N($.$) is a memoryless time-invariant saturation$ nonlinearity. $A \in R^{n \times n}$ is a stable plant matrix, $B \in R^{n \times 1}$ is the input matrix, and $C \in R^{n \times 1}$ is the time-invariant output matrix, and $(\mathrm{A}, \mathrm{B}, \mathrm{C})$ is the minimum realization of the nominal system transfer function:

$$
G(s)=C(s I-1)^{-1} B
$$

The saturation function $N(e)=\operatorname{sign}(1,|k e|)$ belongs to the section $[0, \mathrm{k}]$

$$
0 \leq N(e) \leq k e, \forall t>0, e \in R
$$

where is positive constant, as shown in Fig. 2.

Describing function method (Kandel et al. [8]) marked an important advance in the application of frequency-response

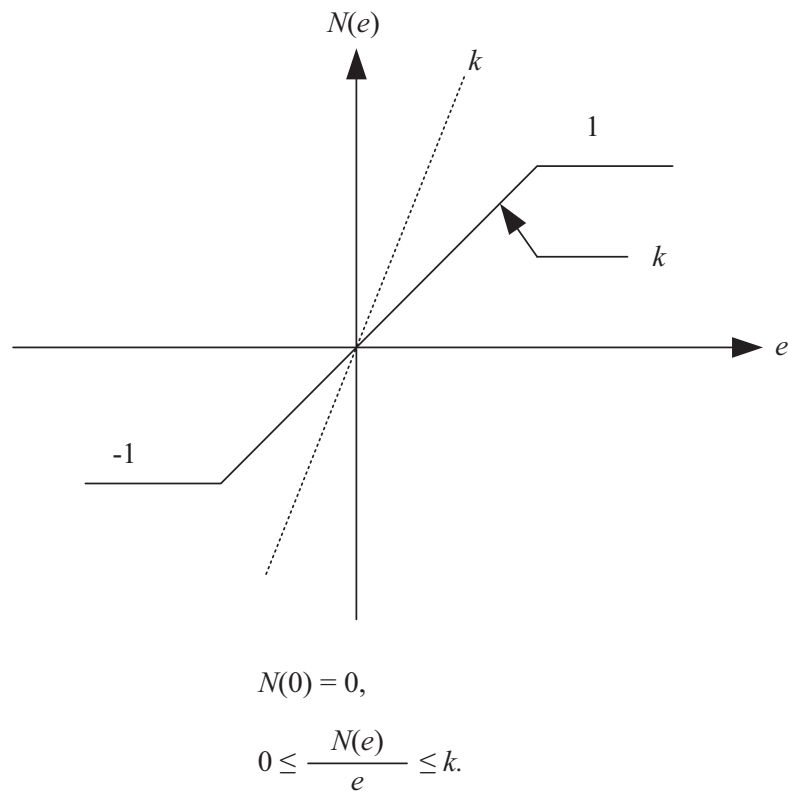

Fig. 2. A scalar nonlinear function $N(e)$ is belongs to sector $[0, k]$.

methods to nonlinear feedback system stability determination since it was a true sufficiency condition for stability. Using this method, the nonlinearity is replaced by its $D F, N(a, \omega)$. The describing function $(D F)$ or sinusoidal describing function of a nonlinear element is defined as the complex ratio of the fundamental harmonic component of the output to the input. If energy-storage elements are included, however, then the describing function is a function of both amplitude and frequency of the input. To determine system stability, plotting $G(j \omega)$ and $-1 / N(a, \omega)$ in the Nyquist plane need to be plotted $N(a, \omega)$ represents the nonlinear controller and $G(j, \omega)$ represents the process under control. The relationship of the saturation of the nonlinearity between input and output can be expresses by the following equations. Assume the input signals to $N(a)$ is $x(t)=a \sin \omega t$, the describing function of saturation can be expressed as [4]

$$
\left.N(a)=2 k / \pi\left[\sin ^{-1}(\delta / a)+(\delta / a) \sqrt{1-(\delta / a}\right)^{2}\right]
$$

For $a>\delta$.

$$
\left\{\begin{array}{l}
a \rightarrow \delta,(\delta / a) \rightarrow 1,-1 / N(a) \rightarrow-1 / k \\
a \rightarrow \delta,(\delta / a) \rightarrow 0,-1 / N(a) \rightarrow \infty .
\end{array}\right\}
$$

Linear stability analysis, using the Nyquist criterion, requires the study of the encirclements of the $(-1 / k, 0)$ point on the real axis by the $G(j \omega)$ locus. In the describing function 


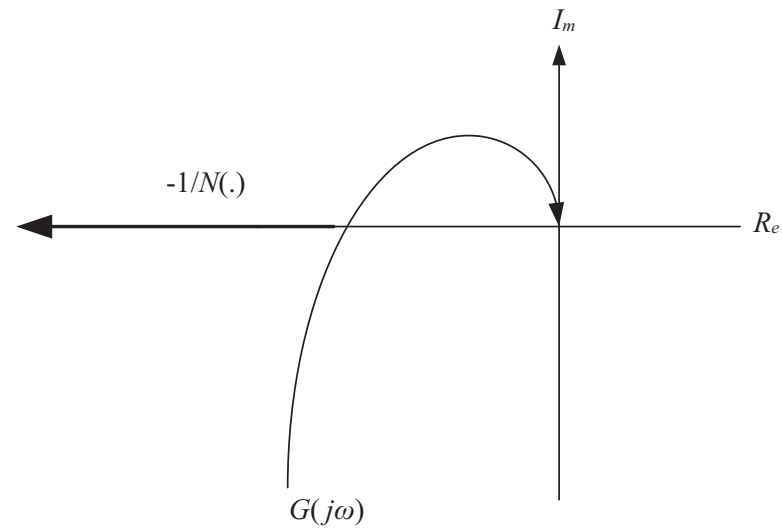

Fig. 3. Detection of system stability by means of $G(j \omega)$ and $\frac{-1}{N(.)}$.

analysis of nonlinear fuzzy control systems, the conventional frequency response analysis is modified so that the entire $\frac{-1}{N(a)}$ locus becomes the locus of critical points. Thus the relative location of the $\frac{-1}{N(a)}$ locus and $G(j \omega)$ locus will provide the stability information. If the $\frac{-1}{N(a)}$ locus is not enclosed by $G(j \omega)$ locus, then the system is stable, or no limit cycle exists at the steady state, as shown in Fig. 3. According to the Nyquist criterion, the linear system with input saturation is absolutely stable if and only if $G(s)$ is Hurwitz and the Nyquist plot of $G(j \omega)$ lied to the right $(-1 / k)$.

$$
R_{e}[G(j \omega)]>-1 / k
$$

Once the plane of the obtained, we can determine the range of the universal discourse for the output of the fuzzy PD system to reduce the overshoot, rise time and oscillation, and to guarantee stability.

\section{DESIGN FUZZY ADAPTATION ALGORITHM}

The proposed fuzzy PD system adaptive tuning proportional controller gain with having input saturation as shown in Fig. 4.

The $k_{p}$ denote the gain of the proportional controller which can be tuned by fuzzy adaptation algorithm. We proposed a stability analysis technique for determining the range $[0, \mathrm{k}]$ of the universal discourse for the output of the fuzzy PD system. Since the output of the fuzzy PD system is the gain $k$ of the proportional controller, the gain $k$ is the range $[0, k]$ of universal discourse of the fuzzy PD system.

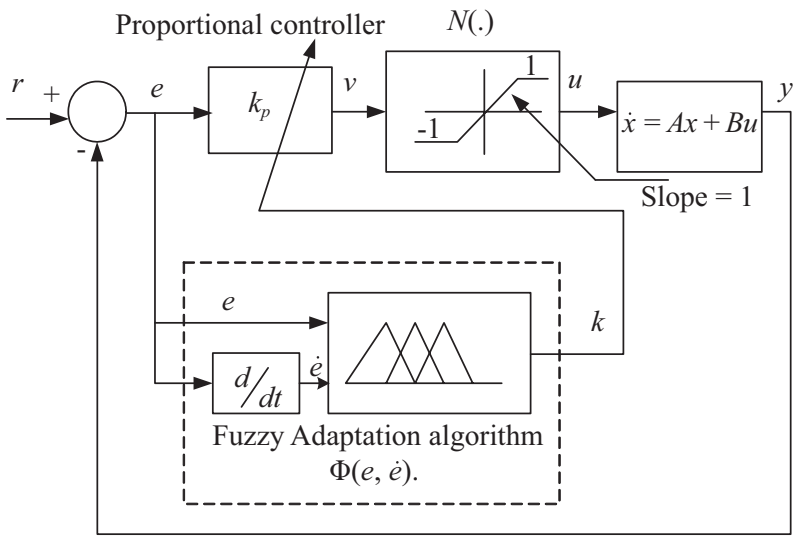

Fig. 4. Proposed fuzzy adaptive control systems having input saturation anti-windup scheme.

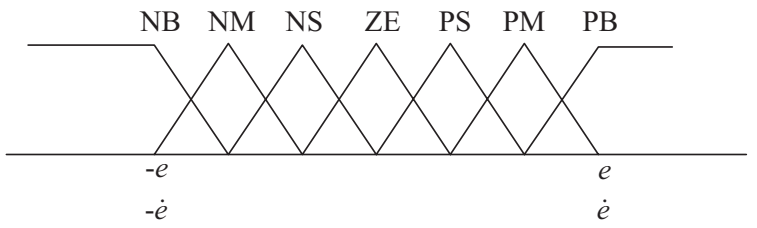

(a) Fuzzy sets for E and DOT_E

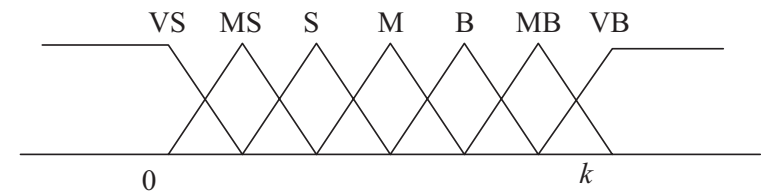

(b) Fuzzy sets for K

Fig. 5. Fuzzy sets and membership functions for E, DOT_E and K.

\section{Application of Fuzzy Adaptive Control System}

The fuzzy adaptation algorithm includes a fuzzification block, a knowledge base, a fuzzy inference engine, and a defuzzification block. The error, $e$, rate of error $\dot{e}$, and $k$ are linguistic variables. Here the error and the rate of change of error are fuzzified and then fed to the fuzzy decision block. The fuzzy inference block refers to the rule-base to generate output fuzzy sets and their membership grades. Then a defuzzification scheme is used to derive the normalized value of the output variable $k$, suppose E, DOT_E and K are linguistic values with membership functions. The label set corresponding to E, and DOT_E can be expressed as follows:

$$
\{\mathrm{NB}, \mathrm{NM}, \mathrm{NS}, \mathrm{ZE}, \mathrm{PS}, \mathrm{PM}, \mathrm{PB}\}
$$

Where each means Negative Big (NB), Negative Medium (NM), Negative Small (NS), Zero (ZE), Positive Small (PS), Positive Medium (PM) and Positive Big (PB). Seven fuzzy sets $\{\mathrm{NB}, \mathrm{NM}, \mathrm{NS}, \mathrm{ZE}, \mathrm{PS}, \mathrm{PM}, \mathrm{PB}\}$ are defined for each input variable $(e$ or $\dot{e})$ with fixed triangular shaped membership functions as shown in Fig. 5(a). The universe of 


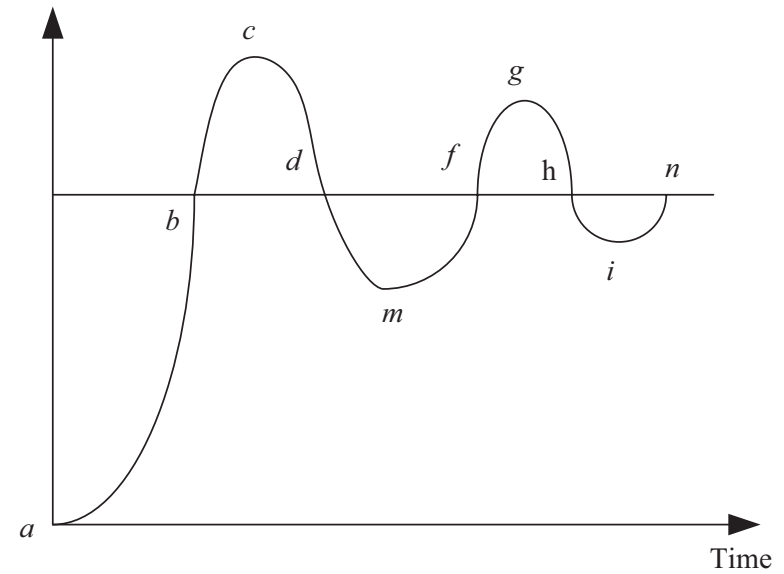

Fig. 6. System step response and phase plane from time domain to rule base.

discourse for E and DOT_E are taken to be in $[-e, e]$ and $[-\dot{e}, \dot{e}]$. For the fuzzy adaptation algorithm, the range of the universal discourse for the inputs of the fuzzy PD system is taken to be in $[-1,1]$. The label set corresponding to $\mathrm{K}$ can be expressed as follows:

$$
\{\mathrm{VS}, \mathrm{MS}, \mathrm{S}, \mathrm{M}, \mathrm{B}, \mathrm{MB}, \mathrm{VB}\}
$$

Where each means Very Small (VS), Medium Small (MS), Small (S), Medium (M), Big (B), Medium Big (MB), Very Big (VB). Seven fuzzy sets $\{\mathrm{VS}, \mathrm{MS}, \mathrm{S}, \mathrm{M}, \mathrm{B}, \mathrm{MB}, \mathrm{VB}\}$ are defined for output variable $k$ with fixed triangular shaped membership functions as shown in Fig. 5(b). The universe of discourse for $\mathrm{K}$ is taken to be in $[0, k]$.

In this study employs the center average method to develop a proportional controller whose gain can be tuned dynamically on-line (i.e. as the controller operates) by the fuzzy adaptation algorithm. The fuzzy adaptation algorithm is constructed by a rule base of individual control rules which are conditional linguistic statements of relationship between inputs and output. The set of control rules can be expressed as follows:

$$
\begin{aligned}
& \mathrm{R}_{\mathrm{i}} \text { : If } e \text { is } \mathrm{E} \text { and } \dot{e} \text { is DOT_E then } k \text { is } \mathrm{K} \\
& \mathrm{R}_{\mathrm{i}} \text { are the fuzzy rules, } i=1,2,3 \cdots, 49 .
\end{aligned}
$$

In fuzzy control region, the error phase plane can act as a bridge between the system performance and the rule base $(\mathrm{Li}$ and Gatland [9]). The fuzzy rules were determined on the characteristic points, $a, b, c, \ldots$ of the general step response curve shown in Fig. 6.

The system equilibrium point is the origin of the phase plane. The set of fuzzy control rules can be determined according to the step response of the system under study. In this study we use a set of fuzzy rules to reduce the overshoot, the rise time, and the oscillation. Importantly, when the output signal is close
Table 1. Rules used in the fuzzy controller.

\begin{tabular}{|c|c|c|c|c|c|c|c|}
\hline NB & NB & NM & NS & ZE & PS & PM & PB \\
\hline NM & VS & VS & VS & MS & S & M & B \\
\hline NS & VS & VS & MS & S & M & B & MB \\
\hline ZE & VS & MS & S & M & B & MB & VB \\
\hline PS & MS & S & M & B & MB & VB & VB \\
\hline PM & S & M & B & MB & VB & VB & VB \\
\hline PB & M & B & MB & VB & VB & VB & VB \\
\hline
\end{tabular}

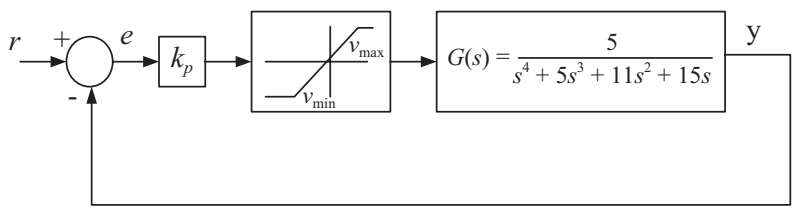

Fig. 7. Equivalent forward transfer functions of control system.

to the set point, the system should have a negative acceleration such that the overshoot tendency is suppressed. In this paper, the used control rules are given in Table 1.

\section{SIMULATION RESULTS}

In order to demonstrate the effectiveness of fuzzy adaptive control system, this study applied the fuzzy adaptation algorithm to the adaptive control system having input saturation.

\section{Example:}

This study considered the problem of the self-guided vehicle system (Nise [10]) of Fig. 7 is operating with a $3 \%$ overshoot.

In this system, the reference input is the desired bearing angle and the output response is the actual bearing angle. Considering the nominal system and the saturation nonlinearity belongs to sector $[0, \mathrm{k}]$. The nominal system transfer function of the system (Nise [10]) is expressed as:

$$
\begin{gathered}
G(s)=\frac{5}{s^{4}+5 s^{3}+11 s^{2}+15 s}, \\
G(j \omega)=\frac{5}{(j \omega)^{4}+5(j \omega)^{3}+11(j \omega)^{2}+15(j \omega)}, \\
G(j \omega)=\frac{5\left[\left(\omega^{4}-11 \omega^{2}\right)-j \omega\left(15-5 \omega^{2}\right)\right]}{\left(\omega^{4}-11 \omega^{2}\right)^{2}+\left[\omega\left(15-5 \omega^{2}\right)\right]^{2}} .
\end{gathered}
$$




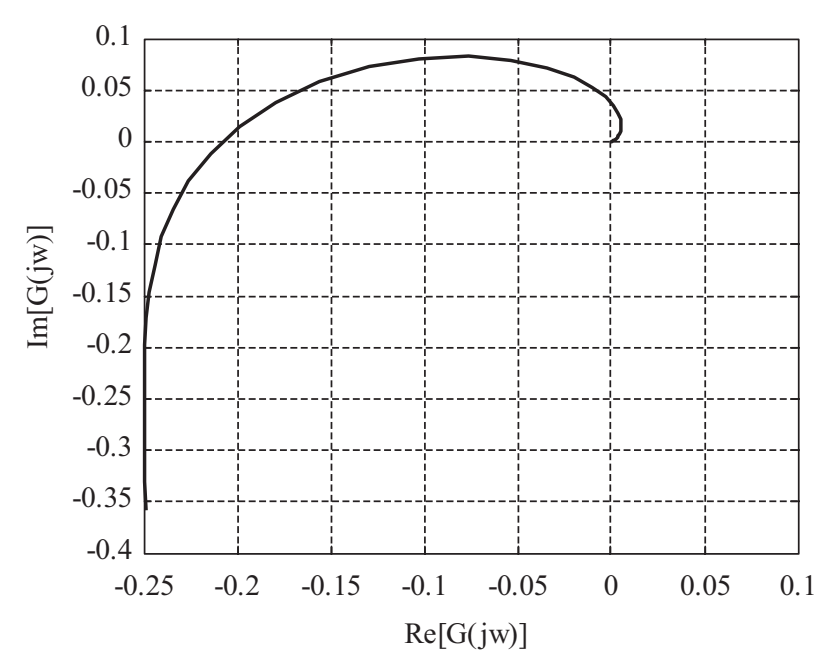

Fig. 8. Nyquist locus of $G(s)=\frac{5}{s^{4}+5 s^{3}+11 s^{2}+15 s}$.

To determine the possible intersection with the real axis, the imaginary part of is set to zero, giving:

$$
I_{m}[G(j \omega)]=\frac{\left.j \omega\left(15-5 \omega^{2}\right)\right]}{\left(\omega^{4}-11 \omega^{2}\right)^{2}+\left[\omega\left(15-5 \omega^{2}\right)\right]^{2}}=0
$$

The solution to (11) is $\omega=\sqrt{3} \mathrm{rad} / \mathrm{sec}$.

Note that for (10):

$$
\begin{gathered}
R_{e}[G(j \omega)]=\frac{5\left[\left(\omega^{4}-11 \omega^{2}\right)\right]}{\left(\omega^{4}-11 \omega^{2}\right)^{2}+\left[\omega\left(15-5 \omega^{2}\right)\right]^{2}}, \\
R_{e}[G(j \sqrt{3})]=-5 / 24,
\end{gathered}
$$

From (6), which is equivalent to the graphical condition that the Nyquist plot of $G(j \omega)$ lies to the right of the vertical line defined by, $R_{e}[G(j \omega)]>-\frac{1}{k}$ the tangential line is found to cross the real axis at -0.21 , as shown in Fig. 8 .

Note that under the Nyquist criterion, the system is stable for $k<\frac{24}{5}$. The range of the universal discourse for the output of the fuzzy PD system is defined to be $\left[0, \frac{24}{5}\right]$.

In this study, MATLAB SIMULINK is used to simulate the control system with a constant reference input.

\section{Combined Back-Calculation and Generalized Conditioning Anti-Windup}

Figure 9(a) shows the block diagram of the conventional PI controller when the parameters are selected based on the Ziegler-Nichols method and the combined back-calculation

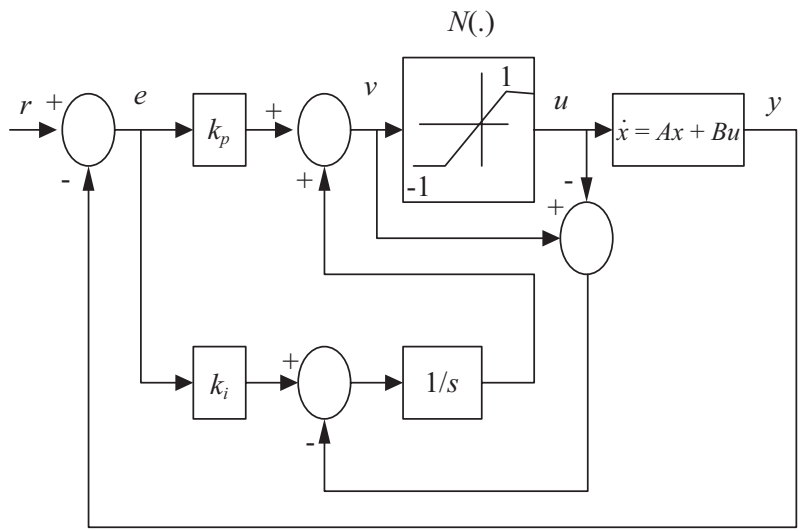

(a)

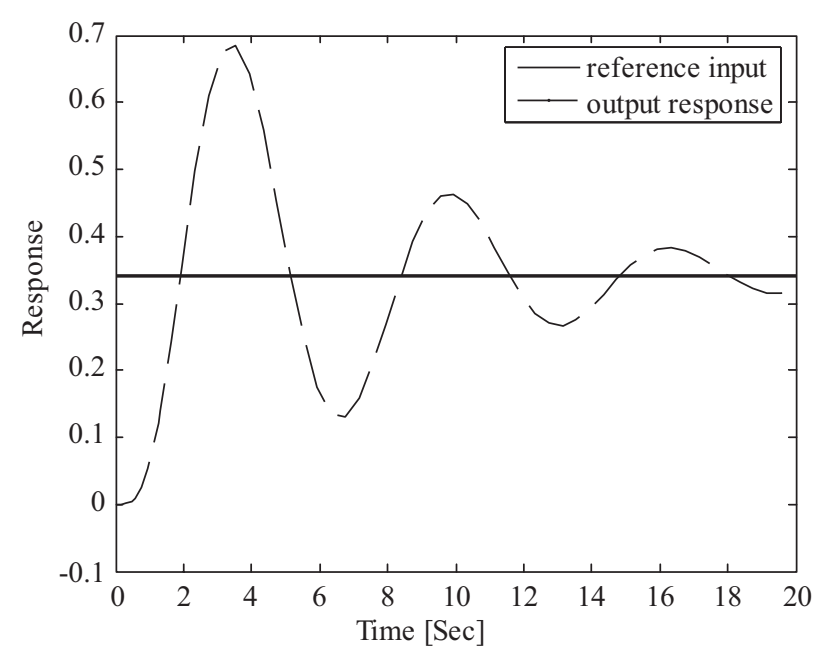

(b)

Fig. 9. Conventional combined back-calculation and generalized conditioning anti-windup scheme. $\left(k_{p}=2.16, k_{i}=0.33, r=0.34\right)$.

Table 2. Performance evaluation results.

\begin{tabular}{|c|c|c|c|}
\hline Peak_of_time & $\begin{array}{c}\text { Percent-overshoot } \\
(\%)\end{array}$ & Rise_time & Setting_time \\
\hline 3.5439 & 101.2783 & 1.0052 & 20 \\
\hline
\end{tabular}

and generalized conditioning anti-windup actuator saturation scheme. Figure 9(b) and Table 2 show the trajectories of the self-guided vehicle's displacement obtained. The "windup phenomenon" degrades the performance of controlled systems, resulting in large overshoots, long settling times.

\section{Proposed Anti-Windup Approach}

The results of Fig. 10 and Table 3 confirmed the superior control performance of the proposed anti-windup adaptive control system.

Furthermore, to test the tracking performance of our approach, three reference input conditions are selected, they are 
Table 3. Performance evaluation results.

\begin{tabular}{|c|c|c|c|}
\hline Peak_of_time & $\begin{array}{c}\text { Percent-overshoot } \\
(\%)\end{array}$ & Rise_time & Setting_time \\
\hline 20 & -0.0511 & 3.7278 & 8.9267 \\
\hline
\end{tabular}

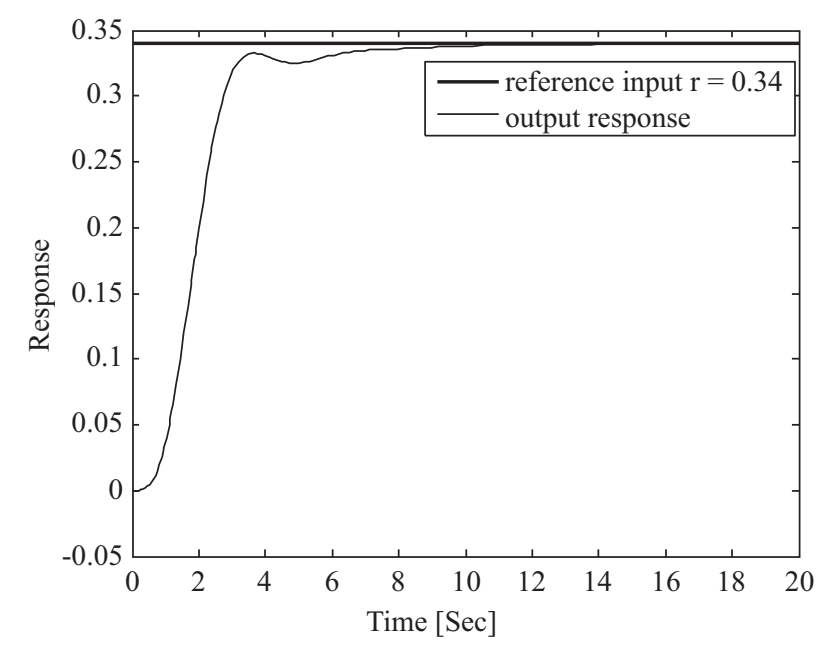

Fig. 10. Responses for proposed anti-windup approach.

(a) Case 1:r $=0.17$; (II) Case 2:r $=1$; (b) Case 3:r $=0.68 \sin$ $0.05 \mathrm{t}$. Figure 11 shows that the adaptive control plant anti-windup system successfully enhances the performance characteristics and improves the tracking performance.

The simulation results demonstrate the successful tracking performance and effectiveness of the adaptive control system having input saturation by the fuzzy adaptation algorithm. Figure 12 shows that the fuzzy adaptive control plant antiwindup system will be used to utilize not only the control error but also the control signal saturation for the modification control input signal. It can be seen that the plant anti-windup adaptive control system not only successfully overcomes the saturation problem, but also improves the performance of the nominal controlled situation.

\section{The LQR Design Method}

We shall now consider the stable control systems based quadratic performance indexes. The block diagram showing the optimal configuration is shown in Fig. 13.

In designing control systems, we are often interested in choosing the control vector $u(t)$ such that a given performance index minimized. It can be proved that a quadratic performance index, such as

$$
J=\int_{0}^{\infty}\left(x^{\prime} Q x+u^{\prime} R u\right) d t
$$

determine the unknown elements of the state feedback matrix $k$ of the optimal control vector
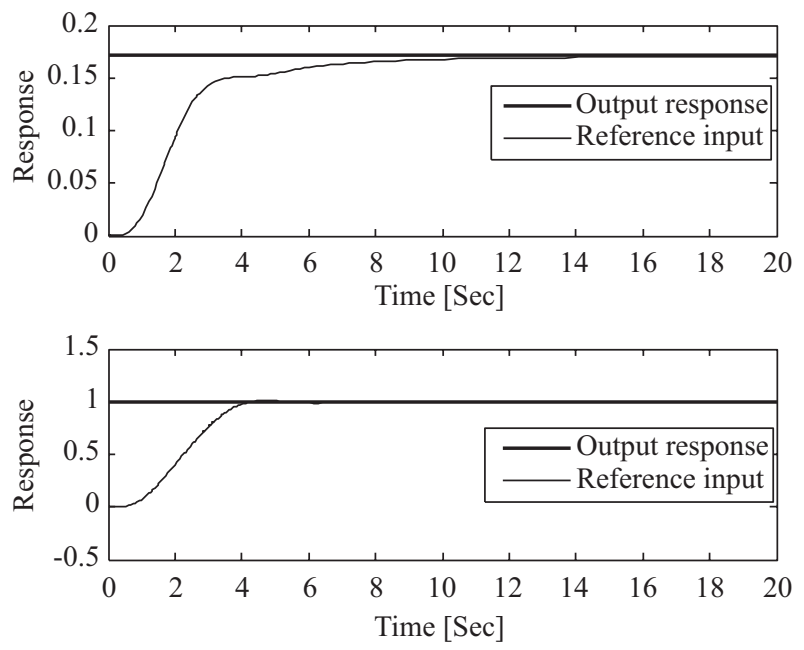

(a)

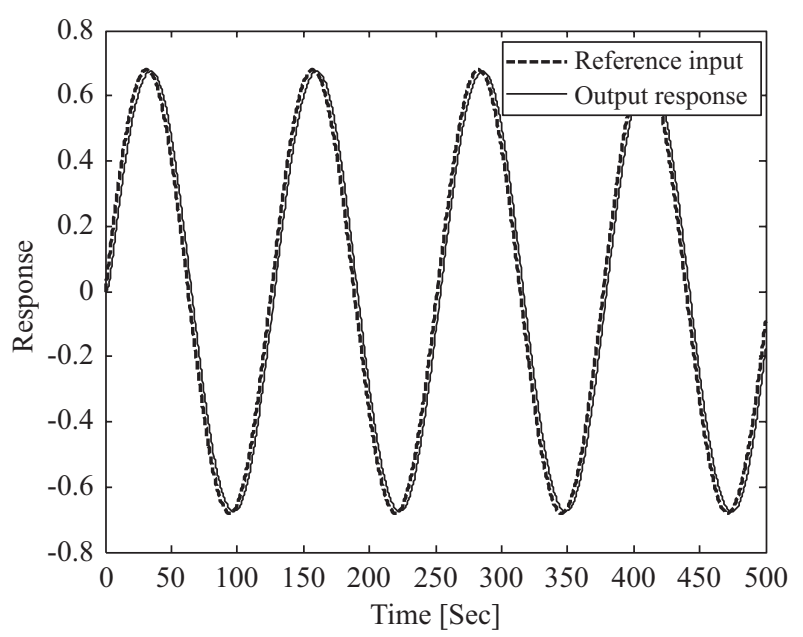

(b)

Fig. 11. Response tracking performance of proposed anti-windup approach.

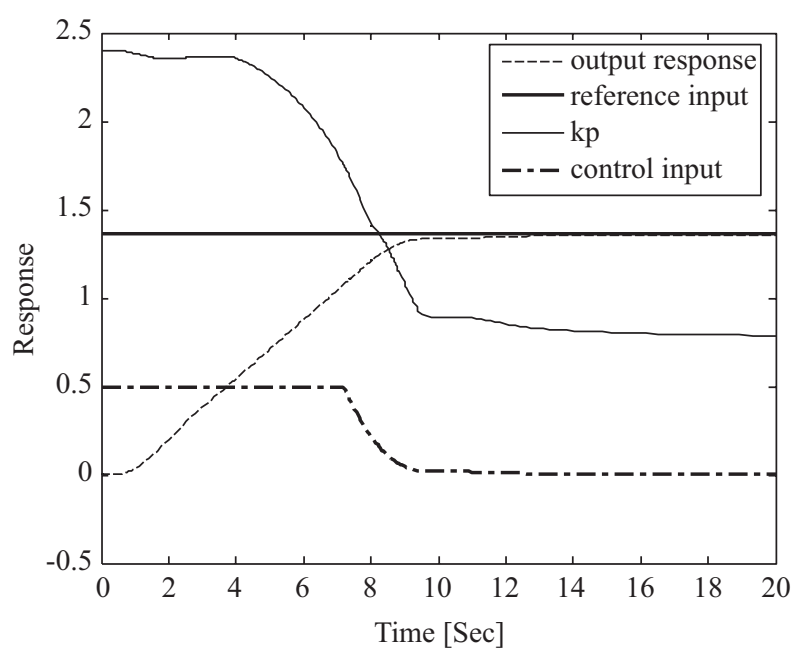

Fig. 12. Response performance of proposed anti-windup approach. 


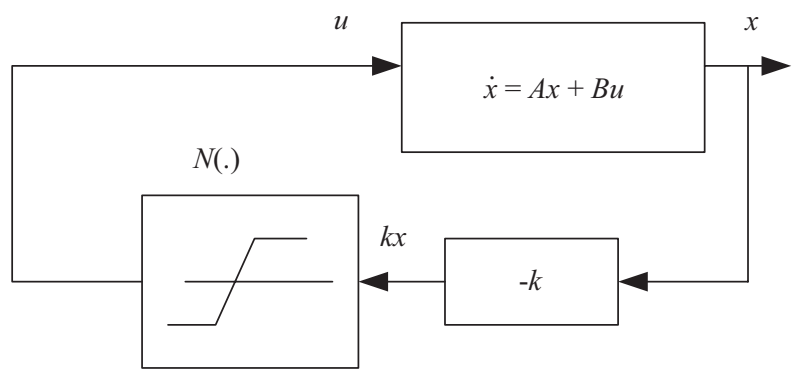

Fig. 13. The block diagram of control system.

$$
u(t)=-N(k x)
$$

where $Q$ is a positive-define (or positive-semidefinite) Hermitian or real symmetric matrix and $R$ is positive- semidefinite Hermitian or real symmetric matrix. Note that the second term on the right side of (13) accounts for the expenditure of the energy of the control signals. Now let us solve the optimization problem. Substituting (14) into (1), we obtain

$$
\dot{x}=A x-B N(k x)
$$

In the following derivations, we assume that matrix $A x-B N(\boldsymbol{k} x)$ is stable or that the eigenvalues of $A x-B N(k x)$ have negative real parts [2]. In MATLAB the command

$$
\boldsymbol{k}=\operatorname{lqr}(A, B, Q, R) \text {. }
$$

solves the continuous-time linear quadratic regular problem and associated Riccati equation. This command calculates the state feedback gain matrix $\boldsymbol{k}$, where

$$
\begin{gathered}
\boldsymbol{k}=\left[\begin{array}{llll}
k_{1} & k_{2} \cdots & k_{r}
\end{array}\right] \\
Q=\left(\begin{array}{cccc}
10 & 0 & 0 & 0 \\
0 & 1 & 0 & 0 \\
0 & 0 & 1 & 0 \\
0 & 0 & 0 & 1
\end{array}\right) \text { and } R=0.01 .
\end{gathered}
$$

From (13), the optimal state feedback gain matrix is found to the

$$
K=\left[\begin{array}{lll}
28.1996 & 38.6062 & 21.292210 .0000
\end{array}\right]
$$

Figure 14 show the response for compared with fuzzy proposed and LQR method with actuator saturation input is implemented.

The "windup phenomenon" degrades the performance of controlled systems, resulting in large overshoots, long settling times. The fuzzy adaptive control system having input satura-

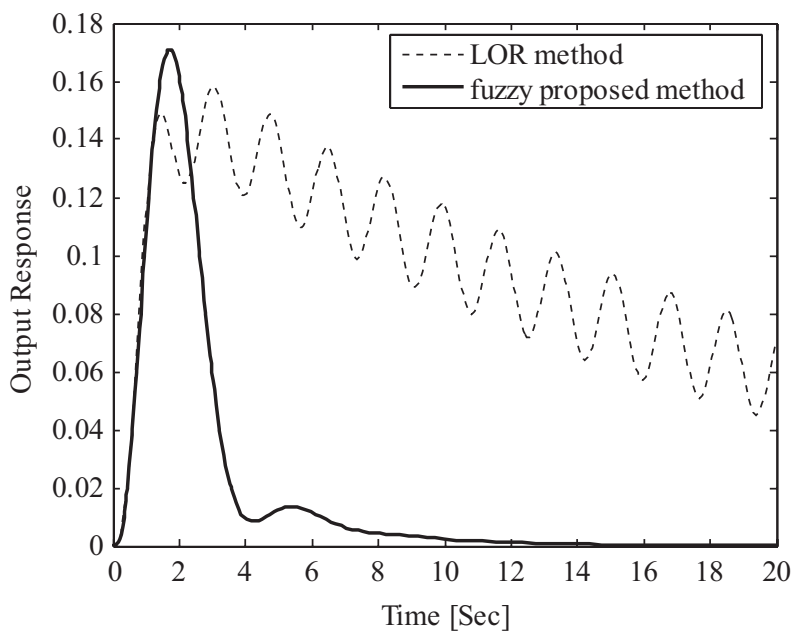

Fig. 14. Responses for compared with fuzzy proposed and LQR method $\left(k_{1}=28.1969, k_{2}=38.6062, k_{3}=21.2922, k_{4}=10.0000\right)$.

tion plant anti-windup ensures system stability and provides a better performance than the LQR method.

\section{CONCLUSION}

This paper has proposed a proportional controller which gain is dynamically tuned by the fuzzy adaptation algorithm to improve the control performance. The simulation results have verified the effectiveness of the proposed plant windup scheme not only successfully overcomes the saturation problem, but also improves the performance of the nominal controlled situation. The fuzzy adaptive control plant anti-windup system will be used to utilize not only the control error but also thecontrol signal saturation for the modification control input signal. The fuzzy adaptive control system having input saturation plant anti-windup ensures system stability and provides a better performance than the LQR method.

\section{ABBREVIATIONS}

A: stable plant matrix

$B$ : input matrix

$C$ : time-invariant output matrix

$e$ : tracking error

$\dot{e}$ : rate of error

$G$ : nominal system transfer function

$N($.): time-invariant nonlinear part

$\boldsymbol{k}$ : state feedback matrix

$k$ : universal discourse for the output of the fuzzy PD system

$k_{p}$ : the proportional gain of the controller

$k_{i}$ : the integral gain of the controller

$Q:$ is a positive-define matrix

$R$ : is positive-semidefinite matrix

$r$ : desired trajectory

$R_{e}$ : real part of the $G(j \omega)$ 
$I_{m}$ : imaginary part of $G(j \omega)$

$u$ : control input

$y$ : output

$\Phi(e, \dot{e})$ : Fuzzy Adaptation algorithm

\section{REFERENCES}

1. Astrom, K. J. and Rundqwist, L., "Integrator windup and how to avoid it," Proceedings of American Control Conference 2, pp. 1693-1698 (1989).

2. Ding, B. C. and Li, S. Y., "Design and analysis of constrained nonlinear quadratic regular," ISA Transactions, Vol. 42, pp. 251-258 (2003).

3. Edwards, C. and Postlethwaite, I., "An anti-windup scheme with closedloop stability considerations," Automatica, Vol. 35, pp. 761-765 (1999).

4. Gelb, A. and Vander Velde, W. E., Multiple input describing functions and nonlinear system design, McGraw-Hill, New York, (1968).

5. Gharieb, W. and Nagib, G., "Fuzzy intervention in PID controller design," Proceedings ISIE, IEEE International Symposium on, Pusan, Korea, Vol. 3, pp. 12-16 (2001).
6. Hansson, A., Gruber, P., and Tödtli, J., "Fuzzy anti-reset windup for PID controllers," Control Engineering Practice, Vol. 2, No. 3, pp. 389-396 (1994).

7. Hippe, P., "Windup prevention for unstable systems," Automatica, Vol. 39, pp. 1967-1973 (2003).

8. Kandel, A., Luo, Y., and Zhang Y. Q., "Stability analysis of fuzzy control systems," Fuzzy set and systems, Vol. 105, pp. 33-48 (1999).

9. Li, H. X. and Gatland, H. B., "Conventional fuzzy control and its enhancement," IEEE Transactions on Systems, Man, and Cybernetics, Vol. 26, pp. $791-797$ (1996).

10. Nise, N. S., Control systems engineering, fourth edition, Wily (2004).

11. Saeki, M. and Wada, N., "Synthesis of a static anti-windup compensator via linear matrix inequalities," International Journal of Robust Nonlinear Control, Vol. 12, pp. 927-953 (2002).

12. Turner, M. C. and Postlethwaite, I., "A new perspective on static and low order anti-windup synthesis," International Journal of Control, Vol. 77, pp. 27-44 (2004).

13. Zhao, Y. and Collins, E. G., "Fuzzy PI control design for an industrial weigh belt feeder," IEEE Transactions on Fuzzy Systems, Vol. 11(1), pp. 311-319 (2003). 